\title{
Antidiabetic effect of Luffa cylindrical extract against the streptozotocin induced diabetes via alteration of glu- cose transporter 2 and sodium-glucose cotransporter 1
}

\author{
Vikas KUMAR $^{*}$, Vastvikta SAHAI
}

Department of Pharmaceutical Sciences, SHUATS, Prayagraj, India

Introduction: Luffa cylindrical [also known as Sponge gourd (SG)] is found to have anti-inflammatory, analgesic, antipyretic, hypoglycaemic, antibacterial, antifungal, antioxidant, anthelmintic, antiviral, anticancer, hepatoprotective, antiemetic, bronchodilator,wound healing, immunologicaland in cataracttreatment. In the following experimental work, we had aimed to estimate the anti-diabetic and hepatoprotective effect of SG methanolicextract in rats which had streptozotocin induced.

Methods: Diabetes in rats was induced by injecting an intra-peritoneal injection of Streptozotocin. Diabetic condition of rats was verified 3 days after Streptozotocin was given, when an elevated blood sugar level was observed in rats. The time duration of our experimental analysis was 28 days. Rats were refrained from eating overnight for oral glucose tolerance test; antioxidant parameters were also reckoned. After 28 days rats were euthanized for conducting histopathological studies.

Results: Depletion in blood glucose level was seen after administering sponge gourd peels extract doses. Influence on HOMA-IR and HOMA- $\beta$, plasma insulin level, glycated hemoglobin and several other parameters was found to be promising. Also, antioxidant parameters, glucose transporter 2 (GLUT2) and sodium-glucose cotransporter 1 (SGLT1) depictedaugmentation in diabetic condition of rats. It can be summarized that methanolic SG extract had a conspicuous effect on Streptozotocin induced rats. Especially $200 \mathrm{mg} / \mathrm{kg}$ dose had stunning results. Upraised levels of GLUT2 and SGLT1 in diabetic rats took the edge off, on treatment with SG extract.

Conclusions: On the basis of result, we can conclude that SG having potential anti-diabetic effect against the STZ induced diabetes. 